follows shortly after nephrotomy-even when the hæmorrhage has persisted for months previously.

Conditions within the kidney causing hæmaturia include calculi. A large branched calculus-the "large silent stone"-rarely produces hæmorrhage. It is the small, more freely mobile stone that does so. The bleeding usually occurs in the next specimen passed after the colic, and the urine contains epithelial cells and sometimes crystals which signify the condition. Occasionally painless hæmaturia is caused by a calculus. Oxaluria may cause considerable hæmaturia. This may supervene after foods rich in oxalates-rhubarb, artichokes, tomatoes, strawberries-or after concentration of the urine by sweating. Associated with the oxaluria there is often scratching of the urethra on micturition, producing pain which is very suggestive. The oxalate crystals occur only in an alkaline urine, and the administration of sodium acid phosphate will lead to a rapid cessation of pain and of hæmorrhage. To prevent occurrence it is essential to drink copiously, and to avoid waters containing much calcium. Also excessive sugar ingestion is unwise, as this can form oxalic acid by bacterial fermentation in the intestines.

Acute pyelitis rarely causes much hæmaturia, but new growths of the pelvis of the kidney, such as papillomata or carcinomata, may do so. A nævoid condition of the apex of the pyramids can be the cause of unexplained hæmaturia, but this is rarely diagnosed apart from exploration.

To locate the cause of hæmaturia may be simple, or on the other hand may require the combined skill of the physician, the surgeon and the pathologist to elucidate a difficult case.

\title{
A CASE OF SILICOSIS.
}

By ERNEST FLETCHER, M.A., M.B., M.R.C.P.

Assistant Physician, Queen Mary's Hospital, Stratford; Medical Registrar, Royal Chest Hospital.

J. B., aged 48 , a fire-bricklayer.

Has had a cough, tightness in the chest, and increasing dyspnœea, for seven years. For the last two years has had occasional streaky hæmoptysis, with abundant foul sputum, and during the last month has had two very large hæmoptyses of a pint each. The weight is steady and the appetite good.

Past History.-No acute illness in the past except an attack of pleurisy one year ago.

Family History.-No history of pulmonary tuberculosis. Fellow-workers suffer from colds and coughs.

On examination, the patient was markedly cyanosed, fingers are clubbed, marked kyphosis.

Chest : "Pigeon-chested," with prominent sternum. Wide intercostal angle, with raising and eversion of lower costal margin.

Heart: A.C.D. goes halt an inch to the right of the sternum, third rib above, the apex beat is in the fifth space half an inch inside the nipple line. Sounds, forcible. Marked epigastric pulsation.

Lungs: Percussion note is resonant over the upper half of the chest, except at the right apex in front. Impaired note at both bases with fine crepitations, universal 
rhonchi, and an area of impaired resonance and bronchial breathing at the right apex in front.

Members were asked to examine this case, and to state their conclusions before the result of any further investigations were given, because it was felt that it should be possible to form a reasonable opinion on the history and physical signs alone.

The long history of a chronic lung infection, with a steady weight, and only occasional fluctuations of temperature (about which very few people asked), should be against tuberculosis, but a great many men assumed the apical cavity to be tuberculous.

The "pigeon chest" was not noticed by most people, so that their attention was not drawn to the kyphosis, and in most cases where it was observed, it was attributed to the lung infection. Kyphosis due to a lung infection is very rare, although scoliosis is a common sequel. Most members said that the patient had emphysema, but few seemed to be able to state their exact reasons for this opinion, i.e., the hyper-resonant note over the upper half of the chest, the wide intercostal angle, the fixed costal cartilages, and the raised everted rib margin. The question as to whether this was likely to be a true bypertrophic emphysema or a compensatory phenomenon was not raised.

Those men who realized that the apical cavity might be bronchiectatic did not appreciate the extreme rarity of apical bronchiectasis, or that apical bronchiectasis without an even greater involvement of the basal bronchi is almost unknown except in certain cases with a specific cause. Few members seemed to be familiar with the fact that bronchiectasis is always a secondary condition, or to be struck by the fact that this chronic lung infection had been progressing for six years before any acute exacerbation had occurred.

That marked epigastric pulsation with a centrally situated heart means enlargemento of the right ventricle did not seem to be generally understood.

It should have been evident so far that here was a man of 49 , with a chronic bilateral lung infection, and a presumably bronchiectatic cavity at the right apex.

Members were now told that repeated sputum examinations had failed to show any tubercle bacilli, and that films and culture showed a mixed flora.

The X-ray picture was typical of an occupational lesion, and the lipiodol filling showed a right upper lobe bronchiectasis with some early dilatation in the right lower lobes.

No member obtained the information which the patient was anxious to give, that his occupation involved the sawing of fire-bricks which contained 80 per cent. silica, although three members asked him whether his occupation was dusty.

Once the diagnosis was established, members seemed to understand the pathology of the condition fairly well, although the close connection of silicosis and pulmonary tuberculosis was not sufficiently realized.

It may be said that this was a difficult case, but its solution really depended on the elementary fact that bronchiectasis is always a secondary condition, and that no case must be put into the general non-specific class until every prime cause has been ruled out.

When the secondary nature of bronchiectasis was emphasized, nearly everyone was able to give several other causes of this condition.

It is probable that the short time available for each case had a great deal to do with the mistakes made. 